\title{
PUMPING OF ROSSBY WAVES AND VORTICES AT THE BASE OF THE SOLAR CONVECTION ZONE
}

\author{
EVGENIY TIKHOMOLOV \\ Institute of Solar-Terrestrial Physics SD RAS, Irkutsk, 664033, \\ P.O. Box 4026, Russia
}

\section{Introduction}

Rossby vortices excited near the base of the solar convection zone are very appealing objects for interpretation of a number of solar phenomena such as long-lived large-scale magnetic structures, the poleward drift of the axisymmetric components after the polar field reversal, and a peculiar long-term behavior of the nonaxisymmetric components (Tikhomolov and Mordvinov 1996).

Rossby vortices can appear due to break down of the Rossby wave with large wavelength (possibly of the order of the solar perimeter) like on the Earth or due to hydrodynamical instability of the zonal flow developed near the base of the solar convection zone. In any case the important issue that should be addressed is the mechanism capable to force large-scale flows in the region under consideration.

\section{The model}

It is supposed that mechanism which pumps large-scale flows operates in high latitudes, near $60^{\circ}$. In that region large-scale flows are in quasigeostrophic regime and some simplifications can be involved. To take into consideration the main specific conditions near the base of the solar convection zone a 2layer model is developed: upper one-third part is convectively unstable, lower two-thirds part are convectively stable. Upper and lower surfaces are nondeformable, impenetrable, stress-free, and have constant temperatures.

The main distinction of the model suggested from the existing models is that the location of the interfacial surface dividing convectively stable and 


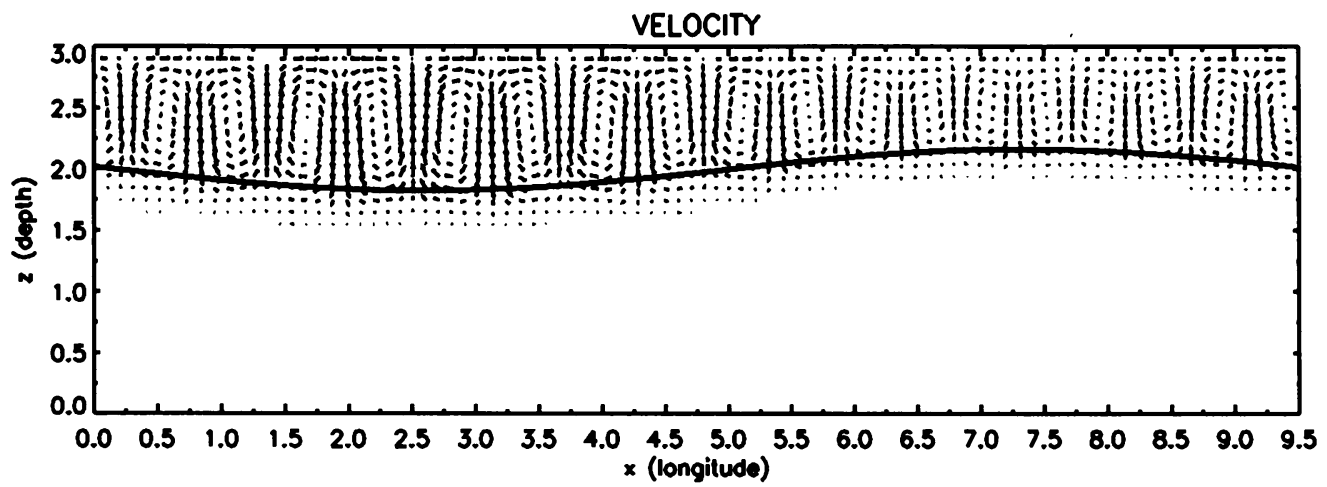

Figure 1. Stationary velocity field established after the initial slight large-scale disturbance of pressure field of stationary convection.

unstable regions slightly varies in depth in accordance with the disturbance of pressure caused by the large-scale flows.

\section{Results}

2D numerical simulations for the model suggested show that stationary convective pattern is in a rather weak equilibrium. Slight disturbance of pressure field by the Rossby wave or by zonal flow with shear in latitude disrupts this equilibrium and amplitude of the flow increases. Nonlinear effects lead to limitation on the amplitude of the flows and stationary picture with disturbed interface is established. This picture for Rossby wave is shown in Fig. 1. Thick solid line indicate the location of the interface dividing convectively stable and unstable regions.

The physical nature of the forcing of the Rossby wave or zonal flow is the appearance of the large-scale temperature distribution dependent on longitude and latitude. The instability mechanism is akin to the one appearing in a rotating single fluid layer heated from below with a deformable upper stressfree surface (Tikhomolov 1994, 1996). So it can also be referred to as the deformational long-wave instability mechanism.

\section{References}

Tikhomolov, E.M. (1994) Sustenance of vortex structures in a rotating fluid layer heated from below, JETP Letters 59, pp. 163-167.

Tikhomolov, E. (1996) Short-scale convection and long-scale deformationally-unstable Rossby wave in a rotating fluid layer heated from below, Phys. of Fluids 8(12), pp. 33293337.

Tikhomolov, E.M. and Mordvinov, V.I. (1996) The peculiar behavior of the large-scale components of the solar magnetic field as a result of Rossby vortex excitation beneath the convection zone, Astrophys. J. 472, pp. 389-398. 